\title{
Randall's plaque of patients with nephrolithiasis begins in basement membranes of thin loops of Henle
}

\author{
Andrew P. Evan, ${ }^{1}$ James E. Lingeman, ${ }^{2}$ Fredric L. Coe, ${ }^{3}$ Joan H. Parks, ${ }^{3}$ Sharon B. Bledsoe, ${ }^{1}$ \\ Youzhi Shao, ${ }^{4}$ Andre J. Sommer, ${ }^{5}$ Ryan F. Paterson, ${ }^{2}$ Ramsay L. Kuo, ${ }^{2}$ and Marc Grynpas ${ }^{6}$
}

\author{
${ }^{1}$ Department of Anatomy and Cell Biology, Indiana University School of Medicine, Indianapolis, Indiana, USA \\ ${ }^{2}$ Methodist Hospital Institute for Kidney Stone Disease, Indianapolis, Indiana, USA \\ ${ }^{3}$ Nephrology Section, University of Chicago, Chicago, Illinois, USA \\ ${ }^{4}$ Department of Histology, Jinzhou Medical College, Jinzhou, Laioning, People's Republic of China \\ ${ }^{5}$ Department of Chemistry and Biochemistry, Miami University, Oxford, Ohio \\ ${ }^{6}$ Samuel Lunenfeld Research Institute, Mount Sinai Hospital, Toronto, Canada
}

\begin{abstract}
Our purpose here is to test the hypothesis that Randall's plaques, calcium phosphate deposits in kidneys of patients with calcium renal stones, arise in unique anatomical regions of the kidney, their formation conditioned by specific stone-forming pathophysiologies. To test this hypothesis, we performed intraoperative biopsies of plaques in kidneys of idiopathic-calcium-stone formers and patients with stones due to obesity-related bypass procedures and obtained papillary specimens from non-stone formers after nephrectomy. Plaque originates in the basement membranes of the thin loops of Henle and spreads from there through the interstitium to beneath the urothelium. Patients who have undergone bypass surgery do not produce such plaque but instead form intratubular hydroxyapatite crystals in collecting ducts. Non-stone formers also do not form plaque. Plaque is specific to certain kinds of stone-forming patients and is initiated specifically in thin-limb basement membranes by mechanisms that remain to be elucidated.
\end{abstract}

J. Clin. Invest. 111:607-616 (2003). doi:10.1172/JCI200317038.

\section{Introduction}

Since he first described them, Randall's plaques have interested researchers who study the pathogenesis of common calcium stones (1). By common calcium oxalate $(\mathrm{CaOx})$ stones, we mean those arising in patients without systemic diseases, apart from those with genetic hypercalciuria (2), who often have relatives with stones. Plaques, defined as sites of interstitial crystal deposition at or near the papilla tip, are found in kidneys of CaOx-stone formers (100\%) (3) and often, but less frequently, in people who do not form $\mathrm{CaOx}$ stones (43\%). Anchored to renal tissue in the renal papillae, and of crystalline composition, they seem an ideal site on which overgrowths of $\mathrm{CaOx}$ or calcium phosphate could grow into stones. In fact, Randall showed examples of $\mathrm{CaOx}$ stones shaped as though they had grown over renal papillae. At the apex of their interior hollow, where the papillary tip would have been, they had patches of calcium phosphate, the same material he found in

Received for publication October 1, 2002, and accepted in revised form December 4, 2002.

Address correspondence to: Andrew P. Evan, Department of Anatomy and Cell Biology, Indiana University School of Medicine, 635 Barnhill Drive, MS 5055, Indianapolis, Indiana 46220, USA. Phone: (317) 274-8102; Fax: (317) 278-2040; E-mail: evan@anatomy.iupui.edu.

Conflict of interest: Fredric L. Coe and Joan H. Parks own stock in LithoLink Corp.

Nonstandard abbreviations used: calcium oxalate $(\mathrm{CaOx})$; Fourier transform infrared microspectroscopy ( $\mu$-FTIR). the plaques themselves (4). In vitro, calcium phosphate phases such as apatite efficiently nucleate CaOx crystallization (5), so that one can easily conjecture that common $\mathrm{CaOx}$ stones begin on plaques.

One major element of such a theory of stone pathogenesis is to know how plaques themselves form: on what structures, and in response to what driving forces. We have hypothesized that plaque is a specialized disease that begins as apatite in a unique region of the kidney because of local driving forces and anatomy. A critical test of this hypothesis is whether or not the most minimal plaque involvement in kidneys of stone-forming patients is always apatite and is always found in one anatomical locale. In other words, does all plaque, however minute, always involve one locale, even if some plaques involve other locales as well? A second hypothesis is that stones that arise from causes radically different from the common $\mathrm{CaOx}$ stone would not necessarily arise on plaque. Consequently, such patients would not have gross plaque and would not have apatite deposits in the regions always involved with plaque in common-stone formers.

Our goal was to test these hypotheses by obtaining biopsies near to and through plaques in the kidneys of patients with renal stones undergoing percutaneous nephrolithotomy, and by studying the tissue using conventional microscopy, electron microscopy, and micro-infrared and $\mathrm{x}$-ray spectroscopy to localize the crystal deposits and determine the crystal type. We present, here, evidence that the most minimal 
plaque lesions are scattered microscopic apatite deposits in the basement membranes of the thin loops of Henle. More extensive plaques extend from there to the papillary interstitium - in some cases, to the regions lying directly beneath the urothelium itself - and are exclusively composed of apatite. Among patients with stones from obesity-related bypass procedures, no plaque is found. Their crystal deposits are not interstitial but arise within tubule lumens and are composed of apatite. Non-stone formers showed no evidence of plaque.

\section{Methods}

\section{Subjects}

We studied 19 patients in each of whom at least two stones had formed. Fifteen fulfilled the criteria for common, so-called idiopathic $\mathrm{CaOx}$ stone formation (6), in that at least one stone was analyzed and shown to be composed of $\mathrm{CaO} x$ and no stones contained uric acid, struvite, cystine, or over $50 \%$ calcium phosphate. None of the 15 had systemic disorders such as primary hyperparathyroidism, sarcoidosis, vitamin D excess, hyperthyroidism, or renal tubular acidosis. As a contrast, we studied four patients whose stones fulfilled the same criteria but who had had intestinal bypass surgery for treatment of obesity and whose stones formed after that surgery. Biopsies were obtained during the course of surgery required for treatment of stone disease. Finally, we studied 4 people ( 1 man) who had no stones nor a family history of stones. Although they had a disease warranting surgery, they serve here as non-stone-forming controls. Two of these patients had renal-cell carcinoma of the clear-cell type. One had a renal angiolipoma, and one had a multilocular benign cyst. In all cases, biopsies were taken from an area remote from the abnormality in question. The two carcinomas were localized within the kidney. Neither of the patients with carcinoma had any evidence of local invasion or metastasis.

\section{Clinical evaluation}

Complete clinical histories were obtained, along with reviews of old records to obtain stone analyses. Two 24-hour urine samples were collected while patients were on a random diet for measurement of volume, $\mathrm{pH}$, calcium, oxalate, citrate, phosphate, uric acid, sodium, potassium, magnesium, sulfate, and ammonia. From these measurements, we calculated supersaturations with respect to calcium oxalate, calcium phosphate (as brushite calcium monohydrogen phosphate), and uric acid using the EQUIL 2 computer program (7). All samples were measured using methods detailed elsewhere (8). Blood was drawn in the fasting state and analyzed for calcium, phosphate, magnesium, sodium, potassium, chloride, creatinine, and carbon dioxide. The blood measurements, not presented here, served to exclude some of the systemic disorders noted above.

\section{Biopsy protocol}

During percutaneous nephrolithotomy performed for stone removal in the patients with idiopathic $\mathrm{CaOx}$ and those who underwent intestinal-bypass surgery, biopsy specimens (9) were taken from one selected papilla in the upper, lower, and interpolar polar regions and from the outer cortex as the working sheath was pulled out of the access site. All biopsy sites were carefully inspected intraoperatively, and none displayed significant hemorrhage. No postoperative complications related to the biopsy process occurred in any patient. Papillary samples were collected from the kidney of each non-stone former after the pathologist had completed a gross examination of the excised kidney. The study was approved by the institutional review board committee for Clarian Health Partners and conducted according to the principles of the Declaration of Helsinki. Each subject gave informed consent.

\section{Tissue analysis}

General analysis. Biopsy and papillary specimens were immediately immersed in 5\% paraformaldehyde in phosphate buffer ( $\mathrm{pH}$ 7.4) and refrigerated for 3 or more days. Forty-three biopsy specimens were studied from the $15 \mathrm{CaOx}$ patients, 19 biopsy specimens from the intestinal-bypass patients, and 9 papillary specimens from the non-stone formers, using light and transmission electron microscopy.

Light microscopic analysis. Individual cortical and papillary biopsy specimens from all $\mathrm{CaOx}$ patients, intestinal-bypass patients, and non-stone formers were dehydrated through a series of graded ethanol concentrations to $100 \%$ ethanol before embedment in a 50/50 mixture of Paraplast Xtra (Fisher Scientific, Itasca, Illinois, USA) and Peel-Away Micro-Cut (Polysciences Inc., Warrington, Pennsylvania, USA). Ten serial sections were cut at $4 \mu \mathrm{m}$ and stained with either the Yasue metal substitution method for calcium histochemistry (10) or hematoxylin and eosin for routine histologic examination. An additional set of serial sections was cut at $7 \mu \mathrm{m}$ for infrared analysis.

Infrared analysis. Tissue sections from 10 of the 15 CaOx-stone formers, from all 4 intestinal-bypass patients, and from 1 non-stone former were analyzed by Fourier transform infrared microspectroscopy ( $\mu$-FTIR) to determine the mineral composition of the sites of crystal deposits. Infrared spectra were collected with a Perkin-Elmer AutoImage infrared microscope interfaced to a Perkin-Elmer Spectrum 2000 Fourier transform spectrometer (Perkin-Elmer, Shelton, Connecticut, USA) in the Molecular Microspectroscopy Laboratory of the Department of Chemistry and Biochemistry of Miami University. The system employed a $250 \times 250$ micrometer, liquid-nitrogen-cooled, mercury cadmium telluride ( $\mathrm{HgCdTe}$ ) detector. Samples were analyzed using a $50 \times 50$ micrometer aperture. Each spectrum represents the average of 64 individual scans possessing a spectral resolution of $4 \mathrm{~cm}^{-1}$. Seven- 


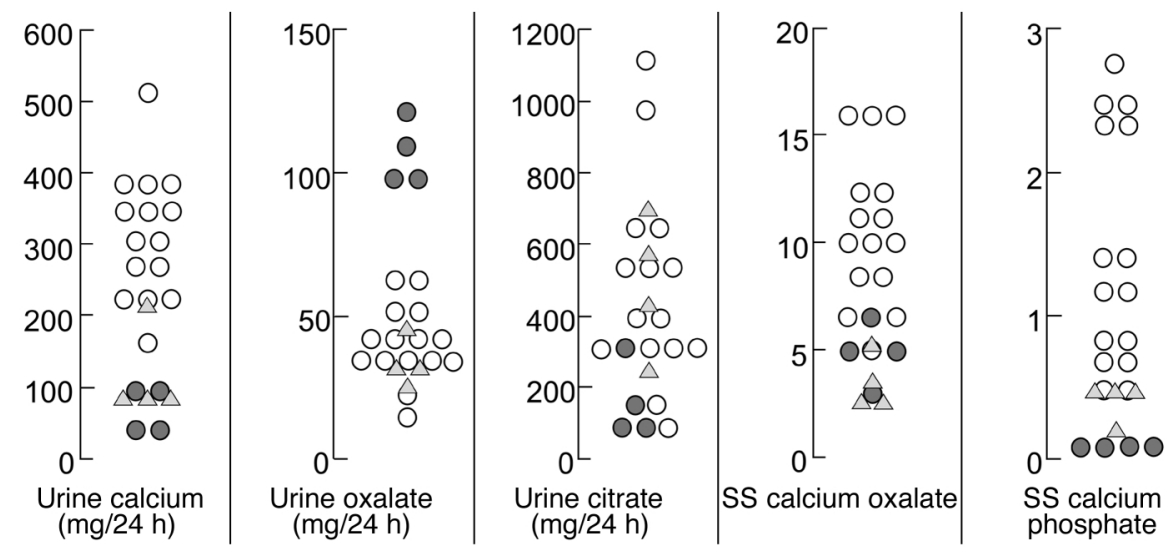

\begin{abstract}
Figure 1
Selected urine values. Common-stone formers are represented by open circles, bypass patients by filled circles, and normal subjects by triangles. The values for a comparison of stone formers with bypass patients are as follows: urine calcium, $312 \pm 89$ versus $81 \pm 29$; oxalate, $40 \pm 13$ versus $106 \pm 11$; citrate, $485 \pm 278$ versus $144 \pm 107$; supersaturation with respect to CaOx monohydrate, $11 \pm 4$ versus $5 \pm 2$; and supersaturation with respect to calcium phosphate, $1.4 \pm 8$ versus $0.09 \pm .05$. $P<0.01$ for all values by $t$ test and Kolmogorov-Smirnov nonparametric testing. For normal subjects, values for calcium, oxalate, citrate, supersaturation with respect to $\mathrm{CaOx}$, and supersaturation with respect to calcium phosphate were $113 \pm 67,32 \pm 9,482 \pm 193,3.5 \pm 1.3$, and $0.42 \pm .16$, respectively; $P=<0.01$ versus stone formers by $t$ test and Kolmogorov-Smirnov for calcium, supersaturation with respect to $\mathrm{CaOx}$, and supersaturation with respect to calcium phosphate; $P<0.0001$ versus bypass by $t$ test and Kolmogorov-Smirnov for oxalate; $P=0.022$ by $t$ test and 0.0001 by Kolmogorov-Smirnov for supersaturation with respect to calcium phosphate; and $P=0.03$ by $t$ test and was not significant by Kolmogorov-Smirnov for citrate. The other comparisons of normal subjects to patient groups were not significant by both $t$ test and Kolmogorov-Smirnov. SS, supersaturation.
\end{abstract}

micrometer-thick paraffin sections were mounted between two barium fluoride salt plates when samples were analyzed in transmission mode. Alternatively, the samples were mounted on a low E-glass slide so that the tissue could be analyzed in the transreflectance mode. This glass transmits visible radiation but reflects infrared radiation. Infrared spectra were collected for standards $(\mathrm{CaOx}$, calcium carbonate, and hydroxyapatite) and regions of tissue with embedding medium for comparative purposes. In this effort to assess the sensitivity of $\mu$-FTIR for the study of the crystal deposits, the relative infrared sensitivities were determined from the absorbance of a given sample with a known thickness. Sensitivities for the oxalate band (1632 band) and the hydroxyapatite band ( 1029 band) were 0.275 and 0.136 , respectively. On the basis of these values, $\mu$-FTIR is approximately two times more sensitive for oxalate than it is for hydroxyapatite. However, due to the presence of an interfering amide I absorption from the tissue located at $1640 \mathrm{~cm}^{-1}$, oxalate was identified by using the 769 $\mathrm{cm}^{-1}$ band. This band has an infrared sensitivity of 0.090 , thereby favoring the detection of hydroxyapatite by a factor of 1.5 .

$X$-ray diffraction. The chemical composition of the crystalline material in the papillary tissue of two $\mathrm{CaOx}$ and two intestinal-bypass patients was determined by standard transmission electron microscopic $\mathrm{x}$-ray diffraction techniques. The analysis was performed with a Philips Tecnai 20 (FEI Co., Eindhoven, The Netherlands).

Transmission electron microscopy. The 5-mm paraformaldehyde-fixed biopsy specimens of the renal papilla were divided into $1-\mathrm{mm}$ blocks, rinsed in phosphate buffer, dehydrated through a series of graded ethanol concentrations to $100 \%$ ethanol, passed through two changes of propylene oxide, and embedded in Epon 812. Thick sections (approximately $1 \mu \mathrm{m}$ ) were stained with toluidine blue, whereas the thin sections (approximately $0.04 \mu \mathrm{m}$ ) were stained with uranyl acetate and lead citrate. All thin sections were examined with a Philips 400 electron microscope.

\section{Statistical analysis}

Each person had two 24-hour urine values. We took the average of the two to make a file with each subject in one row. Routine $t$ tests compared bypass patients to common-stone formers and the normal subjects to

\section{Table 1}

Selected characteristics of the patients

\begin{tabular}{lccc}
\hline & $\begin{array}{c}\text { CaOx-stone } \\
\text { formers (15) }\end{array}$ & $\begin{array}{c}\text { Bypass } \\
\text { patients (4) }\end{array}$ & $\begin{array}{c}\text { Normal } \\
\text { subjects (4) }\end{array}$ \\
& $49 \pm 16$ & $53 \pm 7$ & $51 \pm 15$ \\
(yr) & $103 \pm 29$ & $128 \pm 31$ & $75 \pm 18^{\mathrm{A}, \mathrm{C}}$ \\
ight (kg) & $1.64 \pm 0.6$ & $2.53 \pm 0.28^{\mathrm{B}}$ & $1.86 \pm 0.43^{\mathrm{C}}$ \\
me volume (I/day) & $637 \pm 164$ & $664 \pm 192$ & $585 \pm 178$ \\
acid (mg/day) & $199 \pm 61$ & $342 \pm 43^{\mathrm{B}}$ & $188 \pm 60^{\mathrm{D}}$ \\
ium (mEq/day) & $55 \pm 22$ & $48 \pm 14$ & $59 \pm 18$ \\
assium (mEq/day) & $950 \pm 256$ & $1000 \pm 481$ & $862 \pm 316$ \\
sphate (mg/day) & $117 \pm 36$ & $42 \pm 12^{\mathrm{B}}$ & $60 \pm 17^{\mathrm{B}}$ \\
gnesium (mg/day) & $1673 \pm 370$ & $1594 \pm 384$ & $1449 \pm 811$ \\
atinine (mg/day) & $38 \pm 33$ & $56 \pm 22$ & $34 \pm 16$ \\
monia (mEq/day) & $45 \pm 15$ & $61 \pm 10^{\mathrm{A}}$ & $42 \pm 18$ \\
fate (mEq/day) & $5.89 \pm 0.5$ & $5.57 \pm 0.25$ & $5.94 \pm 0.48$
\end{tabular}

All values $( \pm \mathrm{SD})$ except those for age and weight refer to urine. ${ }^{A} P<0.05$ versus $\mathrm{CaOx}$-stone formers. ${ }^{\mathrm{B} P}<0.001$ versus $\mathrm{CaOx}$-stone formers. ${ }^{\mathrm{C} P}<0.05$ versus bypass patients. $\mathrm{D} P<0.01$ versus bypass patients. 

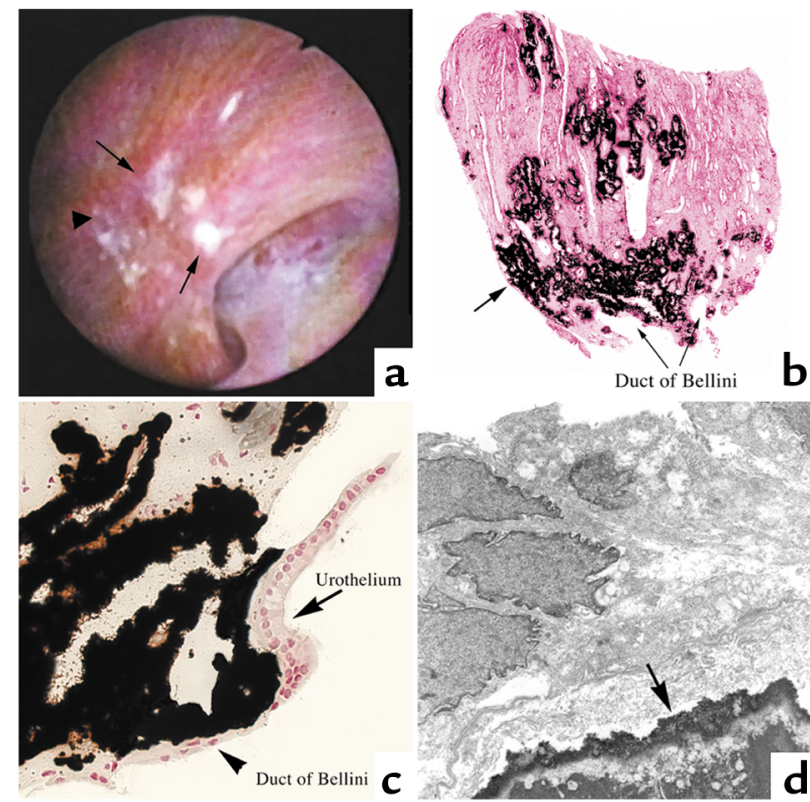

each of the two patient groups. For the most critical values of urine calcium, oxalate, and citrate excretions and supersaturations with respect to $\mathrm{CaOx}$ and calcium phosphate, we confirmed the comparisons using the Kolmogorov-Smirnov nonparametric test.

\section{Results}

\section{Urine data}

Compared with the 4 normal subjects, the $15 \mathrm{CaOx}-$ stone formers had higher urine calcium levels and supersaturations with respect to $\mathrm{CaOx}$ and calcium phosphate (Figure 1), as is usual (11). Compared with the 15 stone formers, the 4 bypass patients excreted less calcium and citrate but more oxalate (Figure 1, filled symbols). Supersaturation with respect to $\mathrm{CaOx}$ was lower among bypass patients, despite high urine oxalate levels, because urine calcium was low and urine volume (Table 1) was high. Calcium phosphate supersaturation was also lower in bypass patients, because of low urine calcium and $\mathrm{pH}$ (Table 1). Compared with the 4 controls, the 4 bypass patients had higher urine oxalate levels, lower supersaturation with respect to calcium phosphate, and marginally lower urine citrate levels. Details of the comparisons are in the figure legends. Overall, both the idiopathic-CaOx-

\section{Figure 3}

Crystalline deposits on interstitial collagen. In (a) and (b), numerous sites of crystal deposition are shown (green arrows) on the interstitial collagen located between the loops of Henle and nearby vascular bundles in the papillary tissue of a $\mathrm{CaOx}$ patient. The inset in (b) illustrates the relationship of the initial small sites of crystal formation on individual collagen fibers at a higher magnification. In (c) and (d), a progressive accumulation of crystal deposition (green arrows) is shown in the interstitium near the loops of Henle. Magnification, $\times 650$ (a); $\times 4,750$ (b); $\times 16,500$ (inset in b); $\times 900$ (c); $\times 3,900$ (d).

\section{Figure 2}

Endoscopic and histologic images of Randall's plaques in $\mathrm{CaOx}$ patients. In (a), an example of a papilla from a CaOx-stone former that was video recorded at the time of the mapping is shown. Several sites of Randall's plaque (arrows) appear as irregular white areas beneath the urothelium in the $\mathrm{CaOx}$ patient. In addition, a plaque site was noted that lacked a urothelial layer and is thought to be a site where a stone had been attached to the side of the papilla (arrowhead). In (b), a low-magnification light-microscopic image of a papillary biopsy specimen from a $\mathrm{CaO}$ patient is shown; the sites of calcium deposits (arrows) were stained black by the Yasue metal substitution method for calcium histochemistry. (c) A light micrograph shows large regions of crystal deposits in the interstitial tissue surrounding the ducts of Bellini (arrowhead), proceeding to the urothelium of the papillary tip (arrow), and progressing up the inner medulla. (d) A transmission electron micrograph shows the crystalline material (arrow) comprising Randall's plaque to be normally covered by a complete layer of urothelium, making the plaque a suburothelial structure. Magnification, $\times 100$ (b); $\times 600$ (c); $\times 6,000$ (d).

stone formers and the bypass patients are much like those we have described in previous reports $(11,12)$.

\section{Histologic data}

Idiopathic $\mathrm{CaO}$ xtone formers. During endoscopy, we identified Randall's plaque in all 15 patients as irregular, whitish lesions, generally located on the papillary tip surrounding the openings of the ducts of Bellini (Figure 2a). Most plaques were suburothelial (deep in the urothelium); the occasional plaque that lacked a urothelial layer (Figure 2a) seemed to be a site where a stone had been attached. Attached stones were removed before biopsy.

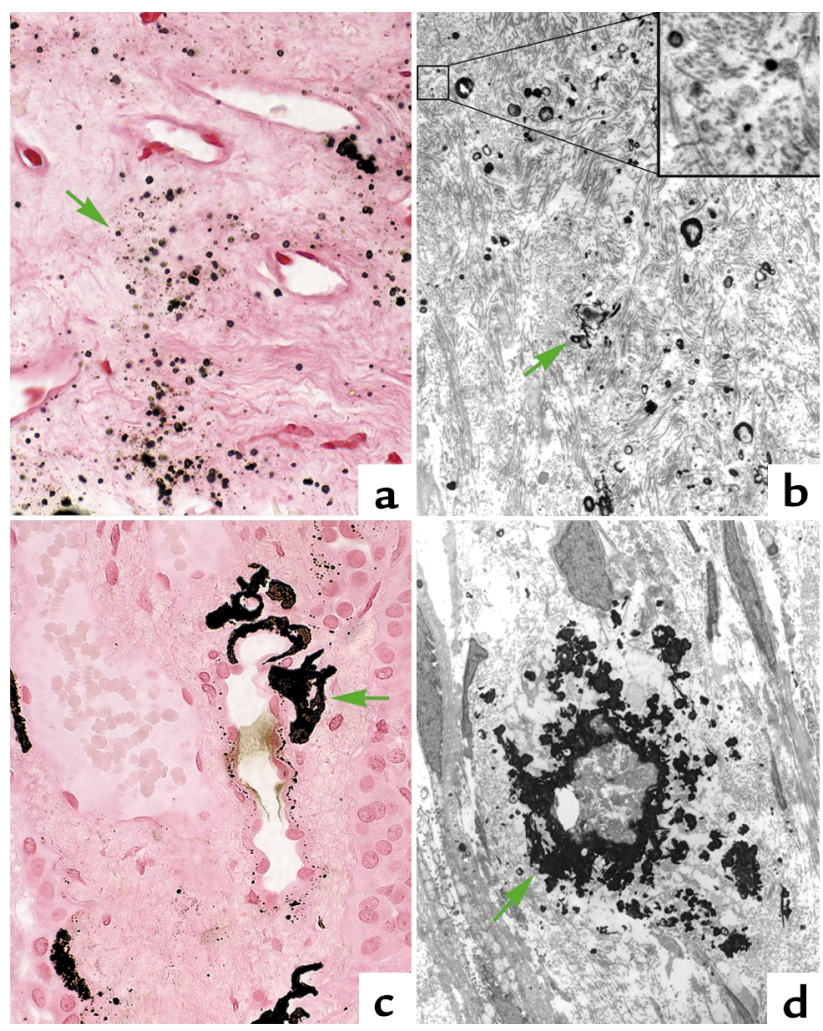



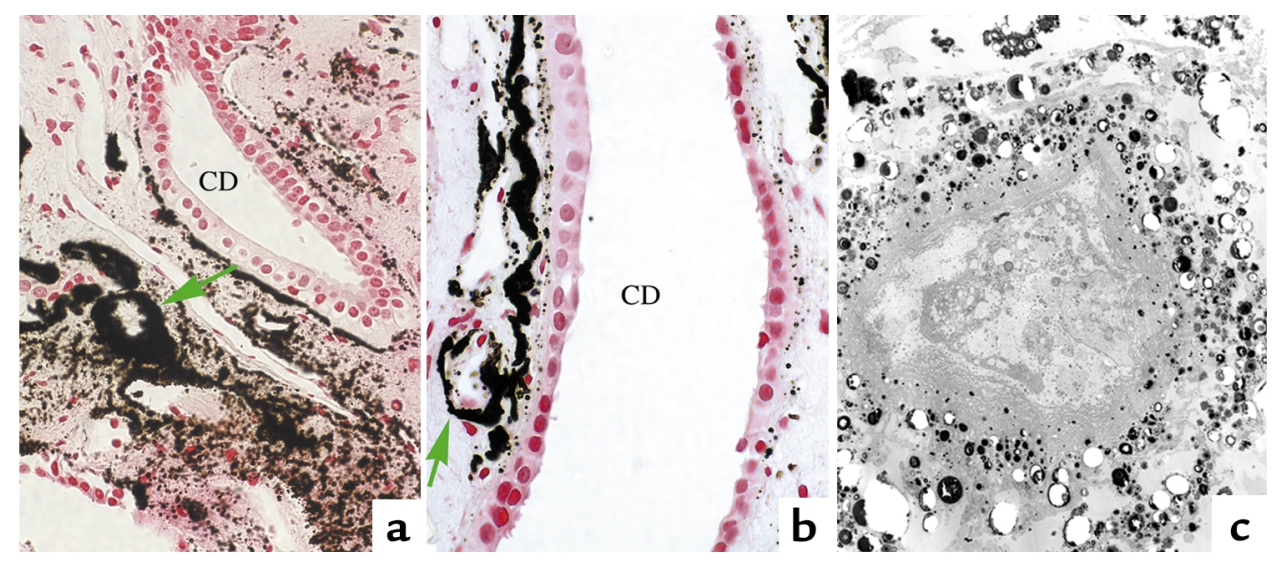

Figure 4

Accumulation of interstitial crystal deposits. In (a) and (b), extensive accumulation of crystalline deposition (green arrows) is shown around a few loops of Henle and nearby vascular bundles, resulting in the formation of incomplete to complete cuffs (indicated by circles) of crystalline material in the papillary tissue of a CaOx patient. Note the accumulation of crystal material in the basement membrane of nearby collecting duct and the normal appearance of the collecting duct cells. In (c), an electron micrograph shows a dense accumulation of crystalline material around a loop of Henle that appears to be necrotic. Magnification, $\times 900$ (a); $\times 1,600$ (b); $\times 400$ (c) . $\mathrm{CD}$, collecting duct.

With low-magnification light microscopy, papillary tissue at sites of suburothelial plaque contained dense regions of deposit just beneath the urothelium (Figure $2, \mathrm{~b}-\mathrm{d})$. The deposits were always located in the interstitial space and followed the bundles of thin loops of Henle and vasa recta up the inner medulla. All 15 patients showed this pattern. Occasionally, crystalline deposits were found not only beneath urothelial cells but between individual urothelial cells (data not shown). No abnormal cellular changes or crystal deposits were noted in the cortical biopsy specimens. At the site of a plaque, interstitial deposits ranged from single spheres to large, dense collections (Figure 3, a-d). As shown by electron microscopy, individual deposits as small as $50 \mathrm{~nm}$ were closely associated with collagen bundles (Figure 3b). Interstitial deposits formed dense rings or bands around some loops of Henle (Figure $3 \mathrm{c}$ ), vasa recta (Figure $3 \mathrm{c}$ ), and interstitial cells (Figure $3 \mathrm{~d}$ ). The dense rings of crystalline material surrounding the loops of Henle varied from incomplete

\section{Figure 5}

Initial sites of crystal deposition. This set of illustrations shows the initial sites and size of calcium deposition in the papillary tissue of a $\mathrm{CaOx}$ patient as seen by light ( $\mathbf{a}$ and $\mathbf{b}$ ) and transmission electron (c and d) microscopy. Sites of crystalline material (arrows) are noted in the basement membranes, near the collagen of the thin loops of Henle (a-c), and to a lesser degree in the basement membranes of vasa recta (d). Magnification, $\times 900$ (a); $\times 1,000$ (b); $\times 15,600$ (c); $\times 5,500$ (d).
(Figures $3 \mathrm{c}, 4 \mathrm{~b}$, and $4 \mathrm{c}$ ) to complete (Figure 4, a-d). Those tubules that were incompletely surrounded by deposits possessed normal-appearing tubular cells (Figure 4a). However, some of the tubules that were completely encased in crystal deposits showed damaged cells, as noted by detachment from their basement membrane and vacuolization of the cytoplasm (Figure $4, \mathrm{c}$ and $\mathrm{d}$ ). In regions where extensive crystal deposi-

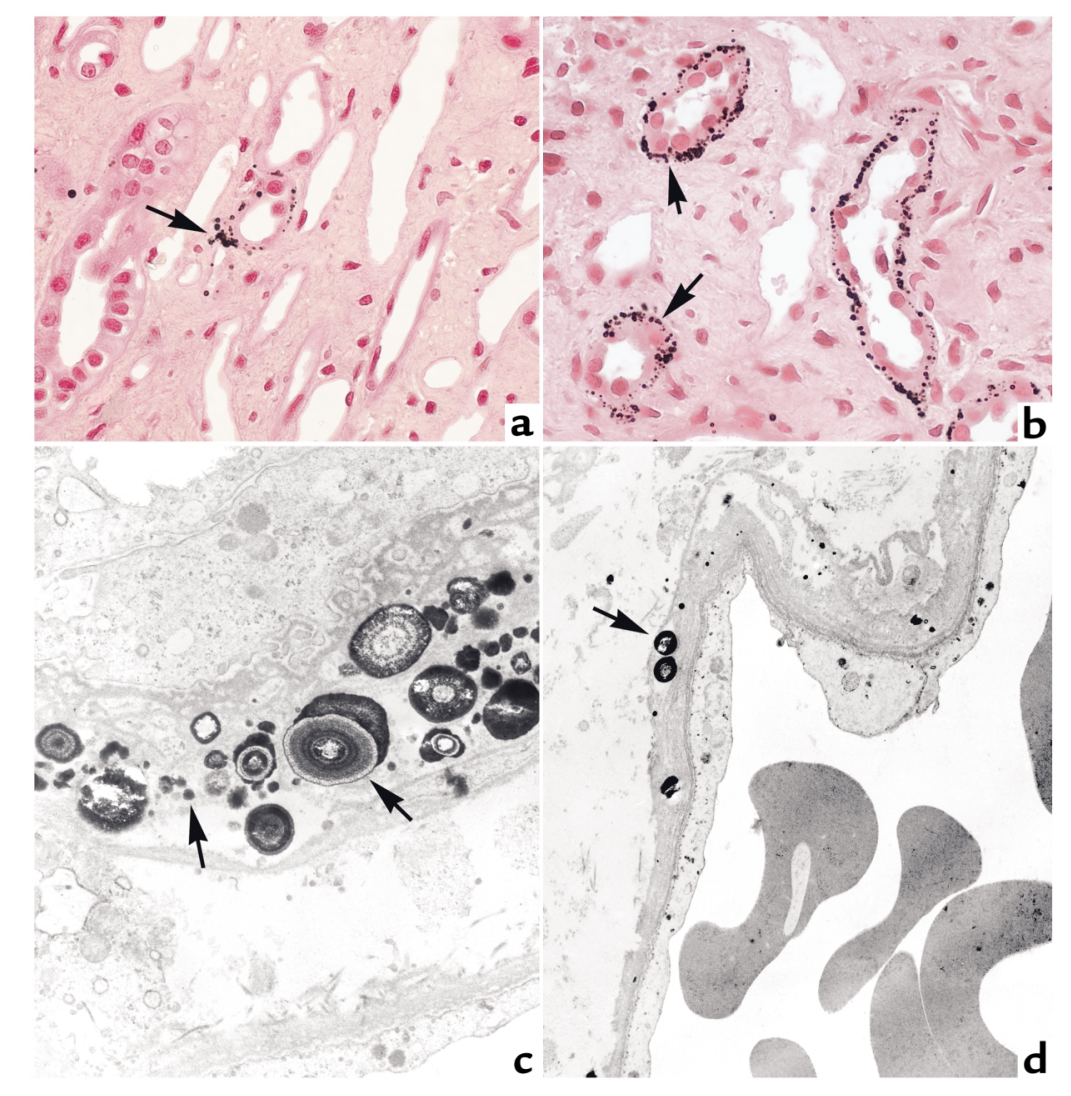



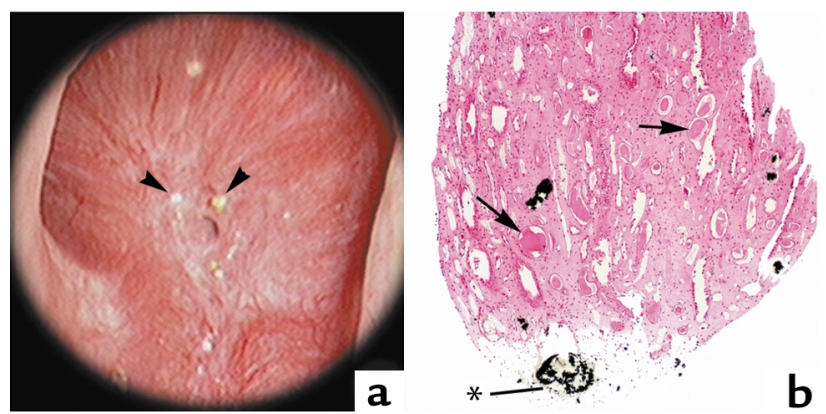

Figure 6

Endoscopic and histologic images of Randall's plaques in intestinal bypass patients. In (a), an example of a papilla from an intestinalbypass stone former that was video recorded at the time of the mapping is shown. Distinct sites of Randall's plaque material are not found on the papilla of the intestinal-bypass patient; instead, several nodular-appearing structures (arrowheads) were noted near the opening of the ducts of Bellini. In (b), a low magnification light microscopic image of a papillary biopsy specimen from an intestinal-bypass patient is shown. Crystal deposition was only found in the lumens of a few collecting ducts as far down as the ducts of Bellini $\left({ }^{*}\right)$. A large site of crystal material was seen in a duct of Bellini. No other sites of deposits were noted. Note dilated collecting ducts (arrows) with cast material and regions of fibrosis around crystaldeposit-filled collecting ducts. Magnification, $\times 100$ (b).

tion involved numerous loops of Henle, crystals accumulated around nearby vasa recta, creating a sleeve of crystal deposition that encased these structures. At the periphery of these dense regions of crystal material were medullary collecting ducts. Occasionally, crystal deposition was noted in the basement membranes of these medullary collecting ducts (Figure 4, a and b); however, the tubule cells always appeared normal. Crystals were rarely found in the cytoplasm of any tubular or vascular cell type.

Although the sites of dense deposits furnished valuable information, they did not allow us to test our hypothesis that crystal deposition would occur at specific sites along the nephron and that these sites would originate as small, discrete apatite deposits. Thus, we examined biopsy specimens of tissue adjacent to regions of Randall's plaques, in order to observe the most minimal sites of crystal deposition. By light microscopy, small Yasue-positive deposits were found surrounding

\section{Figure 7}

Pattern of intraluminal crystal deposition in medullary tissue of bypass patients. In (a), early crystal attachment to the apical surface of several collecting duct cells is shown in a $1-\mu \mathrm{m}$ plastic section. At this stage, these collecting ducts have a mixture of normal and injured $\left({ }^{*}\right)$ cells. In $(\mathbf{b})$, several cells are shown by transmission electron microscopy (see rectangle in a) that possess attached crystals (arrow). In (c), another $1-\mu \mathrm{m}$ plastic section of a collecting duct shows complete crystallization of the tubular lumen and lining cells. In (d), an electron micrograph of a region of the collecting ducts seen in (c) is shown (see rectangle). No cellular detail remains; only crystalline material is found. Magnification, $\times 450(\mathbf{a})$; $\times 6,400(\mathbf{b}) ; \times 550(\mathbf{c}) ; \times 4,000(\mathbf{d})$. primarily thin loops of Henle (Figure 5a). The number of deposits seen around a cross-section of this segment of the nephron varied greatly in number, from 2 to about 40. Also, the number of loops of Henle involved varied from just a single tubular segment (Figure 5a) to multiple segments (Figure $5 b$ ). Occasionally, a few deposits were seen surrounding nearby vasa recta in the less affected areas (Figure $5 \mathrm{~b}$ ). However, the crystal deposits were not associated with the inner medullary collecting ducts (Figure 5a). Transmission electron microscopy showed these deposits to be located within the basement membrane of the loops of Henle and vasa recta; they were spherical in shape and as small as $50 \mathrm{~nm}$ (Figure 5, $c$ and d). The internal features of these deposits varied from a uniform electron-dense appearance to a multilaminated morphology. At these initial sites of crystal deposition, no changes were obvious in the cells of the thin loops of Henle, nor was there evidence of alterations in the nearby interstitial tissues by either light or electron microscopy. No lesion, however small, failed to involve the basement membranes of the thin loops of Henle.

Intestinal-bypass patients. No abnormal cellular changes or crystal deposits were noted in the cortical biopsy specimens. The papillary surface of the intestinalbypass patients, seen at the time of the mapping, showed no Randall's plaque. Instead, small nodular deposits appeared to project off of the urothelium, usually near the openings of the ducts of Bellini (Figure 6a). Histologic examination of the papillary biopsies from these patients revealed Yasue-positive deposits only in the lumens of a few inner medullary collecting ducts as
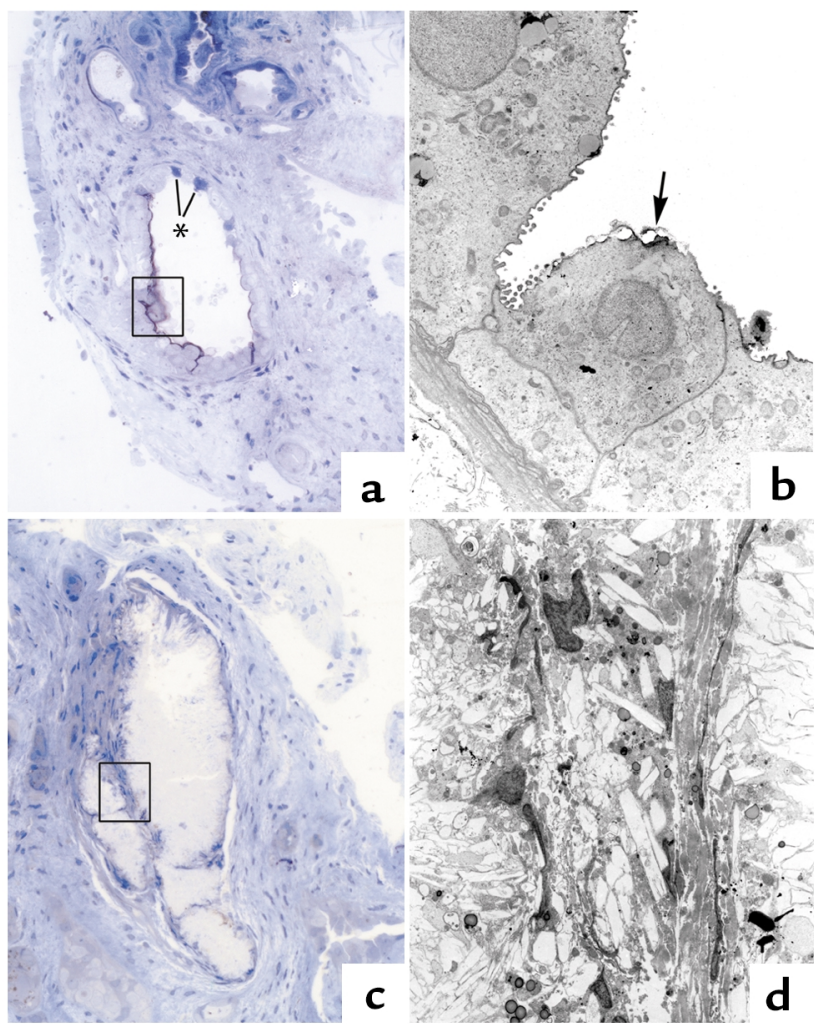

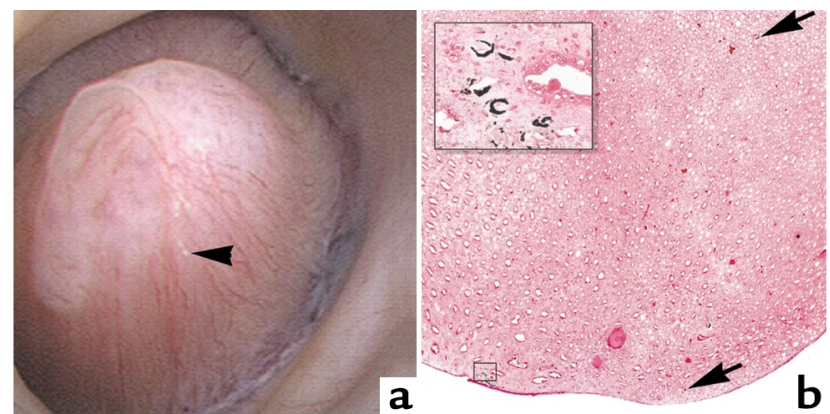

\section{Figure 8}

Endoscopic and crystal deposition in papillary tissue of non-stone formers. In (a), example of a papilla from a non-stone former that was video recorded at the time of the mapping is shown. As in bypass patients, no distinct sites of Randall's plaque are noted on the papilla of the non-stone formers; instead, a nodular-appearing structure (arrowhead) was seen along the side of the papilla. In (b), a low-magnification light-microscopic image of a large papillary sample of the only non-stone former shows several sites of Yasuepositive material. Crystal deposition was only found surrounding a few loops of Henle (arrows) and several vasa recta (inset). Magnification, $\times 15(\mathbf{b}) ; \times 119$ (inset in b).

far down as the ducts of Bellini (Figure 6b). Crystal deposition was not found in the interstitium or surrounding loops of Henle as noted in the $\mathrm{CaOx}$-stone patients.

Crystals were found attached to the apical surface of the collecting duct cells or completely filing the tubular lumen (Figure 7, a and b). At the sites of crystal deposition, the inner medullary collecting duct cells were lined by a mixture of normal and damaged cells. The completely crystallized collecting ducts showed extensive cell injury (Figure 7, $\mathrm{c}$ and d). The intraluminal deposits in such tubules appeared to be obstructing a number of the inner medullary collecting ducts, as evidenced by dilatation of these tubules at and proximal to the obstruction. Many of these upstream dilated collecting ducts contained cast material and were lined by damaged cells. By electron microscopy, the sites of extensive intraluminal crystal deposition revealed a complete loss of all lining cells. In addition, the interstitial tissue surrounding the calcified, dilated collecting ducts was fibrotic in appearance. No sites of crystal deposition were noted in the interstitial tissues.

Non-stone formers. The papillary surface of the non-stone formers, seen at the time of the mapping, showed no Randall's plaques. As in the intestinalbypass patients, small nodular deposits were occasionally noted projecting off of the urothelium (Figure 8a). Histologic examination of the papillary samples did not show any Yasue-positive deposits in two of the non-stone formers, only a single site of Yasue-positive material surrounding a loop of Henle in one non-stone former and several separate sites of Yasue-positive deposits surrounding a loop of Henle or vasa recta in the fourth non-stone former (Figure 8b). In general, the number of Yasue-positive deposits were very few in number considering the much larger tissue samples that were examined for the non-stone formers versus the $\mathrm{CaOx}$ and bypass patients.

\section{$\mu$-FTIR and $x$-ray diffraction analysis}

$\mu$-FTIR analysis of the Yasue-positive deposits in the papillary tissue of all $15 \mathrm{CaOx}$-stone formers detected primary spectral bands for hydroxyapatite (Figure 9). A spectral band for calcium carbonate was also detected at the same sites at which hydroxyapatite was identified in about $20 \%$ of the analysis points. $\mu$-FTIR analysis of the Yasue-positive deposits in the papillary tissue of all four intestinal-bypass stone formers detected primary spectral bands for hydroxyapatite (Figure 9). Similar to the deposits in $\mathrm{CaOx}$ patients, a spectral band for calcium carbonate was also detected at the same sites at which hydroxyapatite was identified in about $10 \%$ of the analysis points. Only hydroxyapatite was detected at sites of deposits in the non-stone formers.

$\mathrm{X}$-ray diffraction analysis identified biological hydroxyapatite (Figure 10, a and b) as the crystal deposition for both the $\mathrm{CaOx}$ and intestinal-bypass patients. However, the $\mathrm{CaOx}$ patients had small, poorly crystalline hydroxyapatite crystals similar to bone, whereas the intestinal-bypass patients had large, plate-like hydroxyapatite crystals similar to those found in kidney stones.

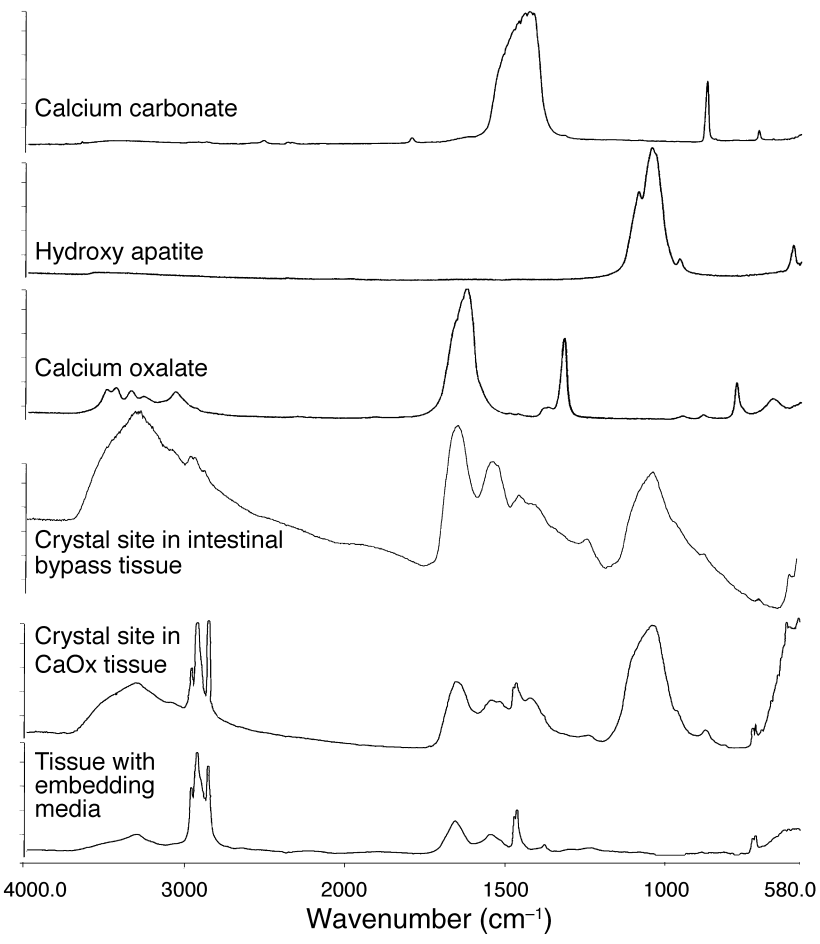

\section{Figure 9}

$\mu$-FTIR spectra of crystal deposits in $\mathrm{CaOx}$ and intestinal-bypass patients. This figure illustrates a series of infrared spectra obtained for a set of standards (calcium carbonate, $\mathrm{CaOx}$, and hydroxyapatite), for a site of calcium deposit (Yasue-positive area) in the tissue of a $\mathrm{CaOx}$ and an intestinal-bypass patient, and for the tissueembedding medium. The infrared spectra of the Yasue-positive area for both the $\mathrm{CaOx}$ and intestinal-bypass patients show a spectral band for hydroxyapatite. 

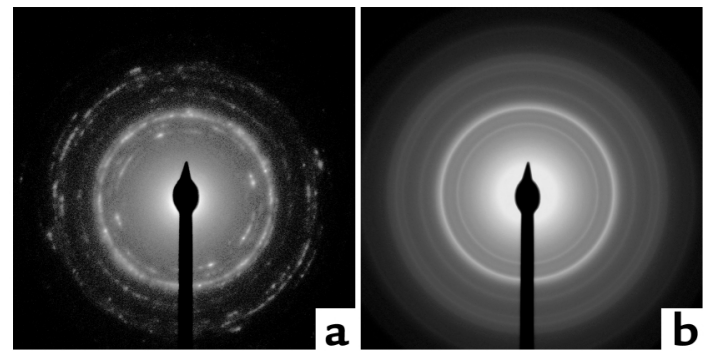

\section{Figure 10}

$X$-ray diffraction patterns of crystalline deposits in $\mathrm{CaOx}$ and intestinal-bypass patients. The crystal deposits in $\mathrm{CaOx}$-stone formers (a) and intestinal-bypass (b) patients were biological hydroxyapatite. However, the deposits were more poorly crystallized in the $\mathrm{CaOx}$ than in the intestinal-bypass patients.

\section{Discussion}

\section{$\mathrm{CaOx}$ stones}

To a great extent, our studies accord with and confirm much that Randall said. Plaque is interstitial and composed of apatite, a structure that he inferred from staining qualities and that we have proven with infrared spectra and x-ray diffraction. What we add, here, is information he could not have easily obtained. As we tracked lesions back to the smallest collections of crystals, even to those visible only by electron microscopy, we found them in the basement membrane of the thin loops of Henle, and even the most minute lesions were apatite. From this point, lesions appear to enlarge, presumably by crystal growth, into the surrounding interstitium and involve the vasa recta. Large lesions reach to the basement membrane of collecting ducts but do not affect the collecting duct cells. Moreover, thin-loop cells also appear completely spared by early lesions. No plaque lesion failed to involve the thin-loop basement membranes. The overall impression is that the basement membrane of thin limbs is indeed the origin of plaque.

We can identify few if any objections to our primary conclusion. It is possible that lesions begin in another anatomical location, spread to the basement membrane of thin limbs, and reabsorb from their sites of origin, leaving only the thin-loop lesions we observe. One would imagine, still, that some biopsy specimens would include crystals that spared the thin-limb basement membrane, but no tissue displays such a property. The same objection holds for the idea that lesions begin as multifocal crystallizations that are preserved in thin-limb basement membrane more than elsewhere and therefore predominate in that location. One would have to assume that all multifocal lesions include thinlimb basement membrane and that the dwell times of lesions elsewhere were indeed so short that we never found lesions that spared the thin-limb basement membrane. The same holds true for the hypothesis that apatite crystals begin within tubule fluid and migrate through thin-loop cells into the basement membranes. We did not find any crystals within cells.
Our placement of the initial plaque lesion in $\mathrm{CaOx}-$ stone formers in the basement membrane of inner medullary thin limbs with normal-appearing cells seems to us entirely original. Randall (9) described a pattern of interstitial crystal deposition (termed type 1) that is consistent with our findings in $\mathrm{CaOx}$-stone formers. Near the papillary tip, crystal deposits surrounded collecting ducts, vasa recta, and loops of Henle. Subsequent studies reported renal papillary calcifications as having numerous origins, including vasa recta, collecting ducts, and/or loops of Henle, and varying degrees of tissue damage (13-20). Of all these studies, only Khan and colleagues (17) have determined the chemical composition of the interstitial deposits, which were hydroxyapatite. The diversity of findings in earlier studies may reflect their being performed on autopsy specimens. The investigators could not obtain adequate 24-hour urinary analyses, nor clinical histories, so they could not classify patients as we have into discrete and reasonably consistent categories. Thus, the consistent patterns of crystal deposition we describe in our well-characterized kidney-stone formers, whose tissue samples were obtained under appropriate fixation conditions, differ from past observations for appropriate reasons.

The basement membrane of thin limbs is not surprising as an initial site for apatite to form. Thin-limb basement membrane is unusually thick and is composed of collagen with considerable mucopolysaccharides (21). Such a matrix offers electrostatically charged ionic sites that can attract calcium and phosphate. Calcium and phosphate anchored on such arrays can form patterns similar to those of crystal lattices, and in this way the protein matrix acts as a template to begin the crystallization process. Once begun in basement membrane, apatite could grow - using calcium and phosphate ions that arrive by diffusion - into the surrounding interstitium, which is rich in collagen and mucopolysaccharides (22). Plaque is therefore an example of crystallization within a collagen matrix that, if it reaches the suburothelial space and penetrates the urothelium, can serve as an anchored site for a $\mathrm{CaOx}$ overlay and, therefore, for $\mathrm{CaOx}$ renal stones.

Judged from micropuncture studies, fluid within the thin segments of the loops of Henle is fully able to initiate calcium phosphate crystallization (23). Solutions modeled in vitro to resemble thin-loop fluid produce an apatite crystal phase. Transmembrane permeabilities for calcium and phosphorus ions for thin loops (23) are very low, but given that loop fluid is produced continuously, it could, at least in theory, load basement membranes with calcium and phosphate and adjust their $\mathrm{pH}$ to a level needed for calcium phosphate crystallization. Since the countercurrent action of the vasa recta limits the efficiency of ion removal, and since the membranes are thick, crystals could form by progressive membrane ion loading. Movement of preformed tubule-fluid apatite into the loop basement membrane is not a good hypothesis, in that 
we found no evidence for crystals in tubule cells, whether the lesions were large or small.

Assuming its origin in the basement membrane of the thin loops of Henle, one might ask why plaque is larger and more common in $\mathrm{CaOx}$-stone formers than in normal subjects, as many have observed before us. Our conjecture is that hypercalciuria is the main reason and acts through multiple mechanisms. Genetic hypercalciuria affects a high proportion of calciumstone formers (6) and acts like a state of vitamin D excess (23-26). Intestinal calcium hyperabsorption is virtually universal and leads to increased postprandial serum calcium levels and filtered loads of calcium (27). Such spikes of high filtered load, and even high blood calcium (about $0.2-0.3 \mathrm{mg} / \mathrm{dl}$ ), would be reflected simultaneously in vasa recta, glomerular filtrate, and fluid presented to the thin limbs. If diet calcium were accompanied by phosphorus, its absorption would also be increased, leading to increased delivery into the deep medulla. High deliveries of calcium to the thick ascending limb of the loop of Henle could increase total calcium reabsorption at that nephron site, enriching the outer medulla with calcium that could be washed into the deep medulla by the descending vasa recta. We present this highly speculative scenario to show plausible means by which hypercalciuria could foster plaque, and we recognize that at each step one needs new experiments as a guide.

\section{Stones from bypass surgery}

Most striking to us, and unambiguous, is the lack of typical plaque in kidneys of the four patients with multiple $\mathrm{CaOx}$ stones ascribed to intestinal bypass. Their papillae show no plaque on biopsy, and their biopsy specimens show no trace of apatite deposits, even by electron microscopy, in the basement membranes of the thin loops of Henle. Instead, their papillae have urothelial nodules that are not interstitial but intratubular. Even more strange, the crystals, as analyzed by both $\mu$-FTIR and x-ray crystallography, are apatite, with no apparent $\mathrm{CaOx}$. We find intraluminal apatite crystals that mainly involve deep papillary collecting ducts, in a pattern similar to the type II deposition described by Randall (9). How apatite crystals form in this site is not clear, as supersaturation of the final urine with respect to brushite, a well-established initial calcium phosphate phase in urine (28-30), was remarkably low (Figure 1). Likewise unclear is why no $\mathrm{CaOx}$ crystals were found given that urine $\mathrm{CaOx}$ supersaturation was present.

We must presume that stones form through an entirely different mechanism in the bypass patients as compared with routine $\mathrm{CaOx}$ stone formers and that their physiology militates against plaque. The latter is not entirely surprising. Calcium absorption by intestine is not increased in bypass patients (31) and is often depressed. Urine calcium excretion by our four bypass patients was, in fact, very low. In addition, we must somehow learn why apatite phases are found in collecting ducts. Clearly, our observations on bypass patients - and the pathophysiology of bypass in relation to apatite formation - require expansion, and all the present work can do is begin the discussion. But even with only four well-studied cases, the absence of any plaque and the presence of intraluminal apatite deposits seem clear signals that the pathophysiology cannot be merely overexcretion with tubule-fluid $\mathrm{CaOx}$ crystallization but is something different. That difference may be the result of an initial obstruction of ducts of Bellini as stones form within the urinary space, which then leads to tubular cell injury and acidification of the tubular fluid as these cells are no longer able to maintain a normal $\mathrm{pH}$ level.

Altogether, as Randall proposed, our findings support the idea that among $\mathrm{CaOx}$-stone formers without systemic diseases or bowel disease, apatite plaque forms and acts as an anchored nucleation site for $\mathrm{CaOx}$. We add to this general formulation that the plaque begins in a unique location, in the basement membrane of the thin loops of Henle, and spreads from there into the papillary interstitium. Renal tubule cells seem uninvolved. The pathophysiology of the initial-site crystallization may well be linked to hypercalciuria. Patients with stones that appear to arise from obesity-related bypass procedures lack plaque altogether, and their peculiar pathophysiology, when compared with that of ordinary stone formers, may well prove to be a key to understanding plaque formation.

\section{Acknowledgments}

This work was supported by NIH grant PO1 DK-56788.

1. Randall, A. 1937. The origin and growth of renal calculi. Ann. Surg. 105:1009-1027.

2. Coe, F.L., Parks, J.H., and Moore, E.S. 1979. Familial idiopathic hypercalciuria. N. Engl. J. Med. 300:337-340.

3. Low, R.K., and Stoller, M.L. 1997. Endoscopic mapping of renal papillae for Randall's plaques in patients with urinary stone disease. J. Urol. 158:2062-2064.

4. Randall, R. 1940. Papillary pathology as precursor of primary renal calculus. J. Urol. 44:580-589.

5. Mandel, G.S., and Mandel, N.S. 1996. Crystal-crystal interactions. In Kidney stones: medical and surgical management. F. L. Coe, M. J. Favus, C. Y. C. Pak, J. H. Parks, and G. M. Preminger, editors. Lippincott-Raven Publishers. Philadelphia, Pennsylvania, USA. 115-128.

6. Coe, F.L., Parks, J.H., and Asplin, J.R. 1992. The pathogenesis and treatment of kidney stones. N. Engl. J. Med. 327:1141-1152.

7. Werness, P.G., Brown, C.M., Smith, L.H., and Finlayson, B. 1985. Equil 2: a basic computer program for the calculation of urinary saturation. J. Urol. 134:1242-1244.

8. Parks, J.H., Goldfisher, E., Asplin, J.R., and Coe, F.L. 2002. A single 24hour urine collection is inadequate for the medical evaluation of nephrolithiasis. J. Urol. 167:1607-1612.

9. Randall, A. 1940. The etiology of primary renal calculus. International Abstract of Surgery. 71:209-240.

10. Yasue, T. 1969. Histochemical identification of calcium oxalate. Acta Histochem. Cytochem. 2:83-95.

11. Parks, J.H., Worcester, E.M., O'Connor, R.C., and Coe, F.L. 2002. Urine stone risk factors in nephrolithiasis patients with and without bowel disease. Kidney Int. In press.

12. Asplin, J.R., Parks, J.H., Nakagawa, Y., and Coe, F.L. 2002. Reduced crystallization inhibition by urine from women with nephrolithiasis. Kidney Int. 61:1821-1829.

13. Burry, A.F., Axelsen, R.A., Trolove, P., and Sallis, J.D. 1976. Calcification in the renal medulla: a classification based on a prospective study of 2261 necropsies. Hum. Pathol. 7:435-449.

14. Haggit, R.C., and Pitcock, J.A. 1971. Renal medullary calcifications: a light and electron microscopic study. J. Urol. 106:342-347. 
15. Vermooten, V. 1942. The origin and development in the renal papilla of Randall's calcium plaques. J. Urol. 48:27-31.

16. Anderson, L.M.J.R. 1946. Origin, frequency and significance of microscopic calculi in kidney. Surg. Gynecol. Obstet. 82:275-282.

17. Khan, S.R., Finlayson, B., and Hackett, R. 1984. Renal papillary changes in patient with calcium oxalate lithiasis. Urology. 23:194-199.

18. Cooke, S.A.R. 1970. The site of calcification in the human renal papilla. Br. J. Surg. 57:890-896.

19. Weller, R.O., Nester, B., and Cooke, S.A.R. 1971. Calcification in the human papilla: an electron-microscope study. J. Pathol. 107:211-216.

20. Stoller, M.L., Shami, G.S., McCormick, V.D., and Kerschmann, R.L. 1996 High resolution radiography of cadaveric kidneys: unraveling the mystery of Randall's plaque formation. J. Urol. 156:1263-1266.

21. Bulger, R.E., and Trump, B.F. 1966. Fine structure of the rat renal papilla. Am. J. Anat. 118:685-696.

22. Pitcock, J.A., Lyons, H., and Brown, P.S. 1988. Glycosaminoglycans of the rat renomedullary interstitium: ultrastructural and biochemical observations. Exp. Mol. Pathol. 49:373-387.

23. Asplin, J.R., Mandel, N.S., and Coe, F.L. 1996. Evidence for calcium phosphate supersaturation in the loop of Henle. Am. J. Physiol. 270:F604-F613

24. Worcester, E.M., and Beshensky, A.M. 1995. Osteopontin inhibits nucleation of calcium oxalate crystals. Ann. N. Y. Acad. Sci. 760:375-377.

25. Adams, N.D., Gray, R.W., and Lemann, J., Jr. 1979. The effects of oral
$\mathrm{CaCO}_{3}$ loading and dietary calcium deprivation on plasma 1,25-dihydroxyvitamin D concentration in healthy adults. J. Clin. Med. Metab. 48:1008-1016.

26. Bushinsky, D.A., Favus, M.J., and Coe, F.L. 1984. Elevated 1,25(OH)2D3, intestinal absorption, and renal mineral conservation in male rats. $\mathrm{Am}$. J. Physiol. 246:F140-F145.

27. Klugman, V.A., and Favus, M.J. 1996. Intestinal absorption of calcium magnesium, and phosphorus. In Kidney stones: medical and surgical management. F. L. Coe, M. J. Favus, C. Y. C. Pak, J. H. Parks, and G. M. Preminger, editors. Lippincott-Raven Publishers. Philadelphia, Pennsylvania, USA. 201-222.

28. Meyer, J.L., Bergert, J.H., and Smith, L.H. 1977. Epitaxial relationships in urolithiasis: the brushite-whewellite system. Clin. Sci. Mol. Med. 52:143-148.

29. Pak, C.Y.C. 1981. Potential etiologic role of brushite in the formation of calcium (renal) stones. J. Cryst.Growth. 53:202-208.

30. Pak, C.Y.C., Eanes, E.D., and Ruskin, B. 1971. Spontaneous precipitation of brushite in urine: evidence that brushite is the nidus of renal stones originating as calcium phosphate. Proc. Natl. Acad. Sci. U. S. A. 68:1456-1460.

31. Worcester, E.M. 1996. Stones due to bowel disease. In Kidney stones: medical and surgical management. F. L. Coe, M. J. Favus, C. Y. C. Pak, J. H. Parks, and G. M. Preminger, editors. Lippincott-Raven Publishers. Philadelphia, Pennsylvania, USA. 883-904. 